Should all adjunctive corticosteroid therapy be avoided in the management of hemodynamically stabile Staphylococcus aureus bacteremia?

Forsblom, E.

2016-03

Forsblom , E , Nurmi , A-M , Ruotsalainen , E \& Järvinen , A 2016 , ' Should all adjunctive corticosteroid therapy be avoided in the management of hemodynamically stabile Staphylococcus aureus bacteremia? ' , European Journal of Clinical Microbiology \& Infectious Diseases, vol. 35 , no. 3 , pp. 471-479 . https://doi.org/10.1007/s10096-015-2563-y

http://hdl.handle.net/10138/161084

https://doi.org/10.1007/s10096-015-2563-y

publishedVersion

Downloaded from Helda, University of Helsinki institutional repository.

This is an electronic reprint of the original article.

This reprint may differ from the original in pagination and typographic detail.

Please cite the original version. 


\title{
Should all adjunctive corticosteroid therapy be avoided in the management of hemodynamically stabile Staphylococcus aureus bacteremia?
}

\author{
E. Forsblom ${ }^{1,2}$ • A.-M. Nurmi ${ }^{1}$ - E. Ruotsalainen ${ }^{1}$ - A. Järvinen ${ }^{1}$
}

Received: 21 October 2015 / Accepted: 16 December 2015 /Published online: 14 January 2016

(C) Springer-Verlag Berlin Heidelberg 2016

\begin{abstract}
The purpose of this study was to examine the prognostic impact of corticosteroids in hemodynamically stabile Staphylococcus aureus bacteremia (SAB). There were 361 hemodynamically stabile methicillin-sensitive SAB patients with prospective follow-up and grouping according to timepoint, dose and indication for corticosteroid therapy. To enable analyses without external interfering corticosteroid therapy all patients with corticosteroid therapy equivalent to prednisone $>10 \mathrm{mg}$ /day for $\geq 1$ month prior to positive blood culture results were excluded. Twenty-five percent (92) of patients received corticosteroid therapy of which $11 \%$ (40) had therapy initiated within 1 week (early initiation) and $9 \%$ (31) had therapy initiated 2-4 weeks after (delayed initiation) positive blood culture. Twenty-one patients $(6 \%)$ had corticosteroid initiated after 4 weeks and were not included in the analyses. A total of $55 \%$ (51/92) received a weekly prednisone dose $>100 \mathrm{mg}$. Patients with early initiated corticosteroid therapy had higher mortality compared to patients treated without corticosteroid therapy at 28 days (20\% vs. $7 \%$ ) (OR, 3.11; $95 \%$ CI, $1.27-7.65 ; p<0.05)$ and at 90 days ( $30 \%$ vs. $10 \%)$ (OR, 4.01; 95\%CI, 1.82-8.81; $p<0.001$ ). Considering all prognostic markers, early initiated corticosteroid therapy predicted 28-day (HR, 3.75; 95\%CI, 1.60-8.79; $p=0.002)$ and
\end{abstract}

E. Forsblom and A-M. Nurmi contributed equally to this work.

E. Forsblom

erik.forsblom@helsinki.fi

1 Division of Infectious Diseases, Inflammation Center, Helsinki University Central Hospital, Helsinki, Finland

2 Division of Infectious Diseases, Department of Medicine, Helsinki University Central Hospital, Aurora Hospital, Nordenskiöldinkatu 26, Building 3, P.O. Box 348, 00029 HUS Helsinki, Finland 90-day (HR, 3.10; 95\%CI, 1.50-6.39; $p=0.002$ ) mortality in Cox proportional hazards regression analysis. When including only patients receiving early initiated corticosteroid therapy with prednisone $\geq 100 \mathrm{mg} /$ week the negative prognostic impact on 28-day mortality was accentuated (HR 4.8, $p=0.001$ ). Corticosteroid therapy initiation after 1 week of positive blood cultures had no independent prognostic impact. Early initiation of corticosteroid therapy may be associate to increased mortality in hemodynamically stabile SAB.

\section{Introduction}

Staphylococcus aureus is one of the most common bacteremic pathogens and associated with considerable mortality of 12 $32 \%$ in recent studies [1,2]. The prognosis of Staphylococcus aureus bacteremia (SAB) is dictated by severe underlying conditions such as immunosuppression and corticosteroid therapy [3-6], severe sepsis and septic shock [4, 7], deep infection foci such as endocarditis $[1,8]$ and methicillinresistance $[1,9]$. Recently, over $70 \%$ of SAB patients have had deep infection foci diagnosed [3, 8].

Hemodynamic instability, i.e. severe sepsis or septic shock, is encountered in 10-38 \% of SAB patients [4, 7]. The role of corticosteroids in the management of severe sepsis and septic shock is continuously debated. Experimental studies have related the potential therapeutic impact of corticosteroids in severe infections to various molecular mechanisms such as prevention of release of interleukin-1 [10] or tumor necrosis factor [11] from mononuclear cells. The first clinical studies in 1976 suggested improved survival with high dose corticosteroid [12] but subsequent randomized-controlled trials failed to confirm these results [13-16]. In the late 1990s and early 2000s prolonged low-dose hydrocortison treatment improved survival in several studies $[17,18]$, including a meta-analysis 
[19]. However, further studies with severe sepsis or septic shock have demonstrated that corticosteroids do not affect 28-day mortality whereas long course treatment with low dose corticosteroids may reduce 28-day all-cause mortality [20], and a recent meta-analysis associated high-dose corticosteroid treatment to an indifferent or higher mortality probability and low-dose corticosteroid treatment to a lower mortality probability [21].

Corticosteroids may be used in hemodynamic stabile SAB patients for various reasons although their impact on patient outcome has not been studied. In bacteremic patients underlying conditions such as rheumatic diseases or chronic pulmonary diseases may necessitate corticosteroid therapy due to exacerbation or non-suitability of other treatment options [4, $6,22-24]$. Corticosteroids may be needed in the management of neurosurgical procedures like subdural hematoma $[25,26]$ in which operative treatment may predispose patients to $\mathrm{SAB}$. There are also SAB-related indications for corticosteroid treatment such as epidural abscesses, to relieve myeloid compression [27], or SAB-induced immune complex glomerulonephritis [28]. It has not been evaluated how common the use of corticosteroids during SAB treatment is and how it affects outcome in hemodynamic stabile patients.

The objective of this study was to investigate the indications, initiation time-point, dose and prognostic impact of adjunctive corticosteroid therapy in hemodynamic stabile $\mathrm{SAB}$ patients. The inclusion of methicillin-sensitive $S$. aureus strains only enabled a study setting without the impact of differences in empirical antibiotic therapy.

\section{Materials and methods}

\section{Settings and study population}

This was a prospective multicenter study including adult patients with at least one positive blood culture for $S$. aureus from five university and seven tertiary care central hospitals in Finland [22]. To enable thorough analyses without any external interfering corticosteroid therapy, all patients with corticosteroid therapy equivalent to prednisone $\geq 10 \mathrm{mg} /$ day for $\geq 1$ month prior to positive blood culture and all patients with severe sepsis or septic shock receiving systemic corticosteroid as part of sepsis therapy at blood culture collection time-point were excluded. Further exclusion criteria were: age $<18$ years, pregnancy, breastfeeding, imprisonment, epilepsy, bacteremia 28 days prior to the study, polymicrobial bacteremia and meningitis [22, 29]. No cases of methicillinresistant $S$. aureus (MRSA) were accepted $(\mathrm{N}=6)$. There was a three-day median time-period between blood culture sampling and study inclusion. Data collection included the following parameters: age, gender, SAB acquisition, underlying diseases and McCabe's classification, length and administration route of antibiotic therapy and any corticosteroid therapy. Documentation of infection foci were based on clinical suspicion or verified by bacteriological, pathological or radiological findings. Laboratory findings, radiological investigations and time to defervescence (axillary temperature below 37.5 ${ }^{\circ} \mathrm{C}$ ) were recorded. Primary endpoint was mortality at 28 or at 90 days. Secondary endpoints were prevalence of deep infection foci and time to defervescence.

\section{Antibiotic therapy}

The standard antibiotic therapy was a semisynthetic penicillin. Patients with contradictions for penicillin were provided with cefuroxime, clindamycin or vancomycin. Furthermore, fluoroquinolone and rifampicin served as additional antibiotic therapy. Length of antibiotic therapy was considered proper when administered intravenously for at least 28 days for a deep infection focus and 14 days in the absence of any deep infection. Detailed information on antimicrobial therapy indications, dosage and administration routes has been provided earlier $[29,30]$.

\section{Follow-up time period}

The prospective follow-up time for the study was 90 days. No patients were lost during the follow-up. Patients that were transferred to other hospitals during the study were followed by direct communication with hospital staff and by thorough patient record retrieval. A follow-up visit at the outpatient policlinic was arranged for each patient who was not hospitalized at 90 days.

\section{Definitions}

Healthcare-associated (HA) SAB was defined as bacteremia with the first positive blood culture for $S$. aureus obtained $\geq 48$ hours after hospital admission or when the patient had remained in a long-term care facility or undergone hemodialysis within the preceding two months. McCabe's criteria were used to classify underlying diseases [31]. Deep infection foci were defined as pneumonia, endocarditis, purulent arthritis, osteomyelitis, deep-seated abscess and foreignbody infections. Severe sepsis was classified as sepsis in combination with hypotension, hypoperfusion, or organ failure and septic shock as sepsis with arterial hypotension despite adequate fluid resuscitation [32].

\section{Corticosteroid therapy}

Corticosteroid therapies were started on a clinical basis and were documented and altered into equivalent doses of prednisone. Therapy was grouped according to (1) onset time-point, (2) cumulative weekly dosage and (3) indication for therapy. 
Initiation time-point was grouped into (i) early initiation (therapy onset within the first week of positive blood culture), (ii) delayed initiation (therapy onset within 2-4 weeks after positive blood culture) and (iii) late initiation (therapy onset past 4 weeks of positive blood culture).

\section{Statistical analysis}

Categorical variables were compared with Pearson's $\mathrm{X}^{2}$ test and non-parametric data were analyzed with Mann-Whitney U-test. Odds ratios (OR) with $95 \%$ confidence intervals (CI) were calculated. Univariate factors with $\mathrm{p}<0.05$ were accepted for Cox proportional hazards regression model analysis of factors predicting mortality, and hazard ratios (HR) with $95 \%$ confidence intervals (CI) were calculated. The Kaplan-Meier method was applied for survival estimates. Analyses were done using SPSS version 12.0 (SPSS Inc., Chicago, IL, USA). All tests were two-tailed and $\mathrm{p}<0.05$ was considered as significant.

\section{Results}

\section{Patient characteristics}

Altogether 430 patients with SAB were included. When accounting for pre-bacteremic corticosteroid use and patients with severe sepsis or septic shock, 69 patients were excluded and 361 hemodynamically stabile methicillin-sensitive SAB patients were accepted for the study (Fig. 1).

\section{Adjunctive corticosteroid therapy}

Ninety-two (25\%) of $361 \mathrm{SAB}$ patients received corticosteroid therapy of whom $40(11 \%)$ had the therapy initiated within one week (early initiation) and 31 (9\%) within 24 weeks (delayed initiation). Furthermore, in 21 (6\%) patients the corticosteroid treatment was initiated after 4 weeks (late initiation) of positive blood culture and these patients were not included in the analyses. Altogether 269 (75\%) patients were treated without corticosteroid therapy (Fig. 1 and Table 1). The weekly mean cumulative corticosteroid dose $( \pm$ standard deviation) was $276 \pm 436 \mathrm{mg}$ prednisone during the first week and weekly corticosteroid doses of at least $100 \mathrm{mg}$ were received by $26 / 92(65 \%)$ patients during the first week and by $12 / 92(39 \%)$ patients during weeks 2 to 4 .

Indications for corticosteroid therapy were recorded from the patient records and in detail analyzed for patients with early initiated corticosteroid therapy (Table 2). In 7 (17\%) patients the indication could not be reliably retrieved. The most common indication was the suspicion or diagnosis of an immunological phenomenon including $30 \%$ of patients, either (i) activation of an earlier diagnosed autoimmune disease (e.g. ancylosing spondylitis), (ii) a reactive process (e.g. reactive arthritis) or (iii) the suspicion of an immunological defect. Among $20 \%$ of patients, early initiated corticosteroid therapy was due to an exacerbation of a pulmonary disease or due to a pleural effusion. In $17 \%$ of cases, corticosteroid therapy was initiated early due to epidural-, para-spinalor pre-sacral abscesses; however, this did not impact mortality. Altogether $6(16 \%)$ patients had corticosteroid therapy initiated early due to purulent pericarditis $(8 \%)$ or due to a neurosurgical procedure ( $8 \%$ ) (Table 2$)$.

The basic characteristics between patients with early, delayed and no corticosteroid therapy are compared in Table 1. No significant differences regarding age, gender, SAB acquisition and chronic diseases according to McCabe's classification were observed except for rheumatoid arthritis which occurred significantly more often among patients with delayed initiation of corticosteroid as compared to no corticosteroid treatment ( $10 \%$ vs. $1 \%, p<0.01)$. However, the total amount of patients with rheumatoid arthritis was only $8(2 \%)$.

\section{Antibiotic therapy}

An intravenous antibiotic therapy effective in vitro against the S. aureus blood isolate was provided to each patient from the day of positive blood culture. Altogether 272 (75\%) patients received anti-staphylococcal penicillin (cloxacillin), 69 $(19 \%)$ received cefuroxime and $8(2 \%)$ received vancomycin (Table 1). No significant difference was observed regarding the distribution of anti-staphylococcal penicillin or cefuroxime between the corticosteroid groups whereas vancomycin therapy was provided significantly more often to patients receiving early $(10 \%)$ or delayed $(6 \%)$ corticosteroid therapy as compared to patients treated without corticosteroid therapy $(1 \%)(p<0.01)$. Clindamycin as antibiotic therapy was received by only 5 ( $1 \%$ ) patients (data not shown). Fluoroquinolone as additional antibiotic was provided to 205 (57\%) and rifampicin to $210(58 \%)$ patients with no significant difference between the corticosteroid groups (Table 1).

\section{Deep infection foci and defervescence}

Altogether 297 (82\%) patients had a deep infection focus diagnosed (Table 1). The vast majority of deep infection foci (76\%) were diagnosed within three days after the positive blood culture and verified by bacteriological, radiological or pathological findings whereas a few pneumonia cases $(\mathrm{N}=5)$ were diagnosed through clinical suspicion only. Patients with early initiated corticosteroid therapy, as compared to patients treated without corticosteroid therapy, had significantly more often pneumonia ( $60 \%$ vs. $30 \%, p<0.001)$ and osteomyelitis (48 \% vs. $32 \%, p<0.05$ ). When comparing patients with delayed initiation of corticosteroid therapy to patients treated without corticosteroid therapy no significant difference was 
Fig. 1 Study profile. Originally, 430 methicillin-sensitive S. aureus bacteremia were recruited. Patients with corticosteroid therapy equivalent to prednisone $\geq 10 \mathrm{mg}$ /day for $\geq 1$ month prior to positive blood culture and all patients with severe sepsis or septic shock were excluded $(\mathrm{N}=69)$. No cases of methicillin-resistant $S$. aureus were included $(\mathrm{N}=6)$. Corticosteroid therapy: Early initiation, i.e. initiation within week 1 of positive blood cultures for $S$. aureus; Delayed initiation, i.e. initiation within week 2-4 of positive blood cultures for S. aureus; Late initiation, i.e. initiation within week $5-12$ of positive blood cultures for S. aureus

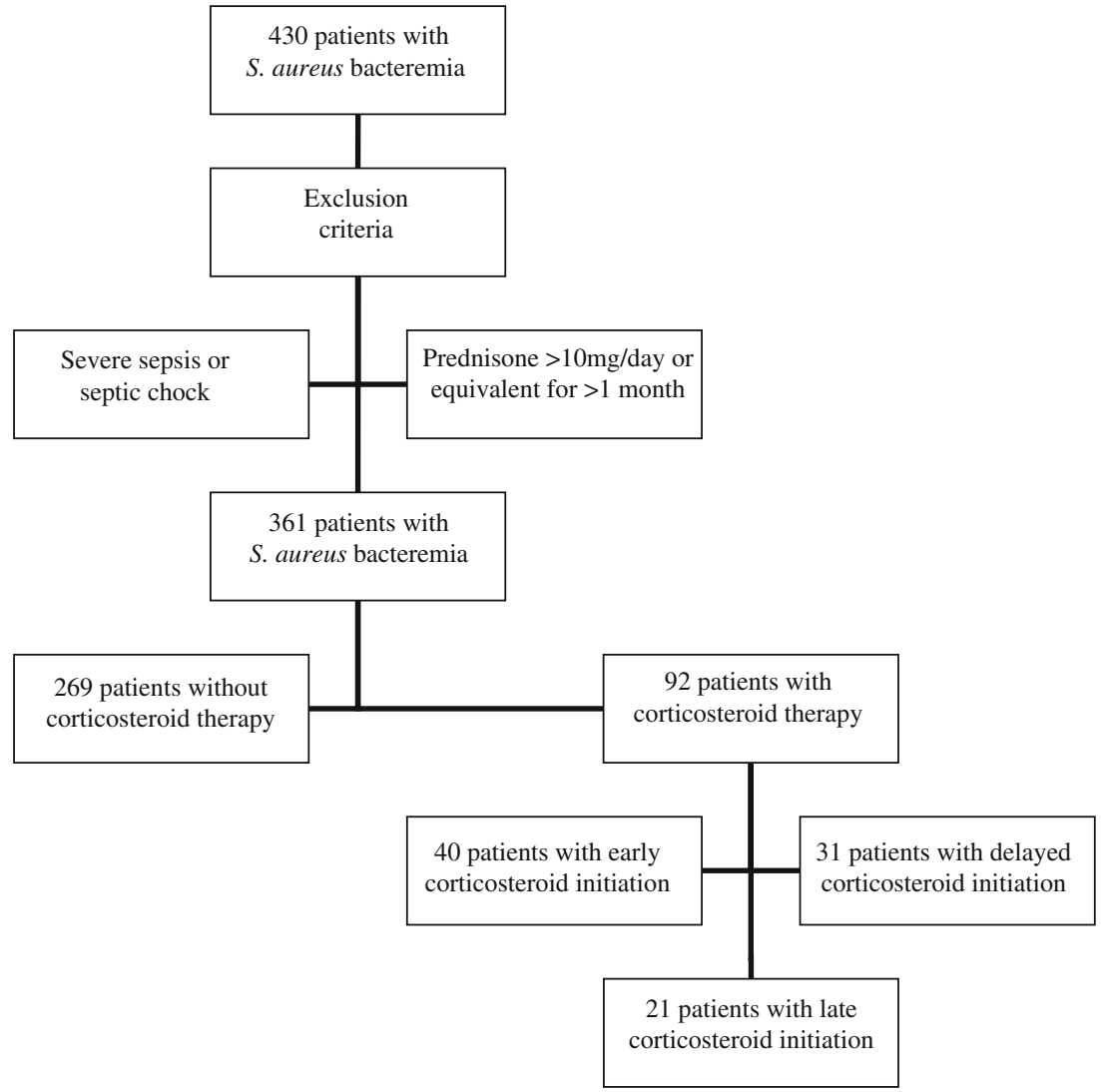

observed in the occurrence of deep infection foci. No differences in invasive eradication of deep infection foci, i.e. abscess punctuation, surgical focus removal or infected joint lavage, were observed between the groups either (Table 1).

\section{Mortality}

No significant differences in time to defervescence were observed between the groups (Table 1). The total case fatality in 361 patients at 28 days was $8 \%$ and at 90 days $12 \%$. Patients with early initiated corticosteroid therapy, as compared to patients treated without corticosteroid therapy, had significantly higher mortality at 28 days $(20 \%$ vs. $7 \%, p<0.05)$ and at 90 days ( $30 \%$ vs. $10 \%, p<0.001)$. No difference in mortality was seen between patients with delayed initiation of corticosteroid therapy and patients treated without corticosteroid therapy (Table 1, Fig. 2).

Mortality was analyzed according to indications for early corticosteroid therapy (Table 2). Patients with corticosteroid therapy initiated due to a complicated pulmonary disease had the highest 90-day mortality (63\%) whereas the corresponding mortality was seemingly lower for patients with corticosteroid due to purulent pericarditis (33\%), craniotomia (33\%) or an immunological phenomenon (17\%). Patients with unrecorded indication for corticosteroid treatment had a high mortality of $43 \%$. No mortality was observed among patients receiving corticosteroid therapy due to epidural-, para-spinalor pre-sacral abscesses (Table 2).

The prognostic impact of early and delayed corticosteroid therapy was evaluated by univariate analysis and Cox proportional hazards regression analysis (Table 3 ). In univariate analysis, parameters for 28-day positive prognostic impact were McCabe's healthy-nonfatal underlying conditions (OR 0.16, $\mathrm{p}<0.001)$ and rifampicin therapy $>14$ days (OR 0.27 , $\mathrm{p}=0.003)$, whereas age $>60$ years $(\mathrm{OR} 11.1, \mathrm{p}<0.001)$, alcoholism (OR 3.52, p=0.003), endocarditis (OR 2.47, p=0.03), pneumonia (OR 2.93, $\mathrm{p}=0.004)$ and early initiated corticosteroid therapy (OR 3.39, $\mathrm{p}=0.005$ ) were found as negative prognostic parameters. Delayed initiation of corticosteroid therapy had no prognostic impact in 28-day univariate analysis. In Cox proportional hazards regression analysis parameters with positive prognostic impact were McCabe's healthynonfatal underlying conditions (HR $0.30, p=0.002$ ) and rifampicin therapy $>14$ days (HR $0.24, p=0.003$ ), whereas age $>60$ (HR 10.1, p<0.001), alcoholism (HR 3.25, $\mathrm{p}=0.008)$, endocarditis (HR 4.49, $\mathrm{p}<0.001)$ and early initiated corticosteroid therapy (HR 3.75, $\mathrm{p}=0.002$ ) were negative prognostic parameters (Table 3 ). When repeating univariate and Cox proportional hazards regression analysis for 90-day prognostic parameters the results resembled those of 28-day prognostic parameters with the exception of chronic renal failure (HR 2.78, p=0.007), malignancy (HR 2.37, p=0.028) 
Table 1 Characteristics of 340 hemodynamically stabile patients with methicillin-sensitive $S$. aureus bacteremia (SAB) categorised according to initiation time-point of adjunctive corticosteroid therapy

\begin{tabular}{|c|c|c|c|c|c|c|c|}
\hline \multirow[t]{2}{*}{ Variable } & \multirow{2}{*}{$\begin{array}{l}\text { No } \\
\text { corticosteroid } \\
(n=269)\end{array}$} & \multirow{2}{*}{$\begin{array}{l}\text { Early } \\
\text { initiation }^{\text {a }} \\
(\mathrm{n}=40)\end{array}$} & \multirow{2}{*}{$\begin{array}{l}\text { Delayed } \\
\text { initiation }{ }^{b} \\
(\mathrm{n}=31)\end{array}$} & \multicolumn{2}{|c|}{ Early initiation vs. no corticosteroid } & \multicolumn{2}{|c|}{ Delayed initiation vs. no corticosteroid } \\
\hline & & & & $\begin{array}{l}\text { OR } \\
(95 \% \mathrm{CI})\end{array}$ & $\mathrm{p}$-value & $\begin{array}{l}\text { OR } \\
(95 \% \mathrm{CI})\end{array}$ & $\mathrm{p}$-value \\
\hline \multicolumn{8}{|l|}{ General characteristics } \\
\hline Age $>60$ years & $126(47)$ & $21(53)$ & $18(58)$ & $1.25(0.65-2.44)$ & NS & $1.57(0.74-3.34)$ & NS \\
\hline Male gender & $168(62)$ & $27(68)$ & $14(45)$ & $1.25(0.62-2.53)$ & NS & $0.50(0.23-1.05)$ & NS \\
\hline Nosocomial bacteremia & $139(52)$ & $16(40)$ & $19(61)$ & $0.62(0.32-1.23)$ & NS & $1.48(0.69-3.17)$ & NS \\
\hline \multicolumn{8}{|l|}{ Underlying diseases } \\
\hline Healthy-nonfatal ${ }^{\mathrm{c}}$ & $208(77)$ & $29(73)$ & $21(68)$ & $0.77(0.37-1.64)$ & NS & $0.62(0.28-1.38)$ & NS \\
\hline Ultimately-rapidly fatal $^{\mathrm{c}}$ & $61(33)$ & $11(28)$ & $10(32)$ & $1.29(0.61-2.74)$ & NS & $1.62(0.73-3.63)$ & NS \\
\hline Rheumatoid arthritis & $4(1)$ & $1(3)$ & $3(10)$ & $1.70(0.19-15.6)$ & NS & $7.10(1.51-33.3)$ & $<0.01$ \\
\hline Connective tissue disease & $11(4)$ & $3(8)$ & $2(6)$ & $1.90(0.51-7.14)$ & NS & $1.62(0.34-7.66)$ & NS \\
\hline Chronic kidney disease & $34(13)$ & $2(5)$ & $5(16)$ & $0.36(0.08-1.58)$ & NS & $1.33(0.48-3.69)$ & NS \\
\hline Chronic lung disease & $37(14)$ & $10(25)$ & $5(16)$ & $2.09(0.94-4.63)$ & NS & $1.21(0.44-3.34)$ & NS \\
\hline Malignancy & $31(12)$ & $6(15)$ & $6(19)$ & $1.36(0.53-3.49)$ & NS & $1.84(0.70-4.84)$ & NS \\
\hline Diabetes & $65(24)$ & $14(35)$ & $5(16)$ & $1.69(0.83-3.43)$ & NS & $0.60(0.22-1.64)$ & NS \\
\hline HIV-infection & $8(3)$ & 0 & 0 & - & - & - & - \\
\hline Injection drug use & $31(12)$ & $5(13)$ & $2(6)$ & $1.10(0.40-3.01)$ & NS & $0.53(0.12-2.33)$ & NS \\
\hline \multicolumn{8}{|l|}{ Antibiotic therapy } \\
\hline Cloxacillin $^{\mathrm{d}}$ & $206(77)$ & $27(68)$ & $23(74)$ & & NS & & NS \\
\hline Cefuroxime $^{\mathrm{d}}$ & $55(20)$ & $5(13)$ & $4(13)$ & & NS & & NS \\
\hline Vancomycin ${ }^{\mathrm{d}}$ & $2(1)$ & $4(10)$ & $2(6)$ & & $<0.01$ & & $<0.01$ \\
\hline Fluoroquinolone ${ }^{\mathrm{C}}$ & $154(57)$ & $20(50)$ & $21(68)$ & & NS & & NS \\
\hline Rifampicin $^{\mathrm{e}}$ & $154(57)$ & $23(58)$ & $17(55)$ & & NS & & NS \\
\hline Rifampicin $>14$ days $^{\text {e }}$ & $124(46)$ & $15(38)$ & $14(45)$ & & NS & & NS \\
\hline \multicolumn{8}{|l|}{ Infection diagnostics } \\
\hline Any deep infection focus ${ }^{\mathrm{f}}$ & $210(78)$ & $38(95)$ & $28(90)$ & $5.34(1.25-22.8)$ & $<0.05$ & $2.62(0.77-8.93)$ & NS \\
\hline Pneumonia ${ }^{\mathrm{f}}$ & $82(30)$ & $24(60)$ & $13(42)$ & $3.42(1.73-6.78)$ & $<0.001$ & $1.65(0.77-3.52)$ & NS \\
\hline Endocarditis ${ }^{\mathrm{f}}$ & $39(14)$ & $5(13)$ & $8(26)$ & $0.84(0.31-2.28)$ & NS & $2.05(0.86-4.91)$ & NS \\
\hline Septic arthritis ${ }^{\mathrm{f}}$ & $33(12)$ & $6(15)$ & $5(16)$ & $1.26(0.49-3.24)$ & NS & $1.38(0.49-3.83)$ & NS \\
\hline Osteomyelitis ${ }^{\mathrm{f}}$ & $85(32)$ & $19(48)$ & $11(35)$ & $1.96(1.00-3.83)$ & $<0.05$ & $1.19(0.55-2.60)$ & NS \\
\hline Any deep abscess ${ }^{\mathrm{f}}$ & $113(42)$ & $22(55)$ & $16(52)$ & $1.69(0.87-3.29)$ & NS & $1.47(0.69-3.10)$ & NS \\
\hline Abscess drainage & $67(25)$ & $15(38)$ & $11(35)$ & $1.81(0.90-3.63)$ & NS & $1.66(0.76-3.64)$ & NS \\
\hline Surgical focus removal & $59(22)$ & $13(33)$ & $11(35)$ & $1.71(0.83-3.53)$ & NS & $1.96(0.89-4.32)$ & NS \\
\hline Infected joint lavage & $8(3)$ & 0 & 0 & - & - & - & - \\
\hline \multicolumn{8}{|l|}{ Outcome } \\
\hline Time to defervescence ${ }^{\mathrm{g}}$ & $4.32 \pm 5.39$ & $4.15 \pm 6.12$ & $4.35 \pm 3.16$ & - & & - & NS \\
\hline Mortality within 28 days & $20(7)$ & $8(20)$ & $2(6)$ & $3.11(1.27-7.65)$ & $<0.05$ & $0.86(0.19-3.86)$ & NS \\
\hline Mortality within 90 days & $26(10)$ & $12(30)$ & $3(10)$ & $4.01(1.82-8.81)$ & $<0.001$ & $1.00(0.29-3.52)$ & NS \\
\hline
\end{tabular}

Originally, altogether $430 \mathrm{SAB}$ patients were identified. Patients with corticosteroid therapy equivalent to prednisone $\geq 10 \mathrm{mg} / \mathrm{day}$ for $\geq 1 \mathrm{month}$ prior to positive blood culture and all patients with severe sepsis or septic shock receiving systemic corticosteroid as part of sepsis therapy at blood culture collection time-point were excluded $(\mathrm{N}=69)$. Twenty-one patients had corticosteroid therapy initiated 4 weeks past positive blood culture and these were not included in the analysis. Data are no. (\%) of patients unless otherwise specified. NS non-significant

${ }^{\text {a }}$ Corticosteroid therapy initiation within week 1 of positive blood culture for $S$. aureus

${ }^{\mathrm{b}}$ Corticosteroid therapy initiation within week 2-4 of positive blood culture for $S$. aureus

c According to McCabe and Jackson [31]

d Standard antibiotic therapy

e Additional antibiotic therapy

${ }^{\mathrm{f}}$ Diagnosed within 90 days time

$\mathrm{g}$ Time given in days (mean \pm SD) 
Table 2 Categorization of 40 hemodynamically stabile patients with $S$. aureus bacteraemia (SAB) according to indication for adjunctive corticosteroid therapy started within a week after positive blood culture

\begin{tabular}{lllll}
\hline $\begin{array}{l}\text { Indication for } \\
\text { corticosteroid use }\end{array}$ & $\begin{array}{l}\text { Patient number } \\
(\mathrm{n}=40)\end{array}$ & $\begin{array}{l}\text { 3-day } \\
\text { mortality }\end{array}$ & $\begin{array}{l}\text { 28-day } \\
\text { mortality }\end{array}$ & $\begin{array}{l}\text { 90-day } \\
\text { mortality }\end{array}$ \\
\hline $\begin{array}{l}\text { 1. Purulent pericarditis } \\
\text { 2. Craniotomy }\end{array}$ & $3(8)$ & 0 & 0 & $1(33)$ \\
3. Epidural, para-spinal or pre-sacral abscess & $3(8)$ & 0 & 0 & $1(33)$ \\
4. Complicated pulmonary disease a $^{\text {a }}$ & $7(17)$ & 0 & 0 & 0 \\
5. Activation of autoimmune disease & $8(20)$ & 0 & $5(63)$ & $5(63)$ \\
6. Other indication & $12(30)$ & 0 & $1(8)$ & $2(17)$ \\
\hline
\end{tabular}

Data are number (\%) of patients

${ }^{\text {a }}$ Chronic obstructive pulmonary disease, asthma bronchial or pulmonary obstruction.

${ }^{\mathrm{b}}$ Earlier diagnosed autoimmune disease and delayed initiation of corticosteroid therapy (HR 2.32, $\mathrm{p}=0.014$ ) associating to negative prognosis in univariate analysis (data not shown).

As a further investigation, we performed a Cox proportional hazards regression analysis including only patients with chronic pulmonary disease or SAB related pneumonia $(\mathrm{N}=164)$. The results of this analysis closely resemble those of Table 3 with a 90-day negative prognostic impact due to early initiated corticosteroid therapy (HR 2.94, $\mathrm{p}=0.008$ ). This prognostic impact was further accentuated when including only patients receiving early initiated corticosteroid therapy with a minimum of prednisone $100 \mathrm{mg} /$ week (HR 4.65, $\mathrm{p}<0.001)$.

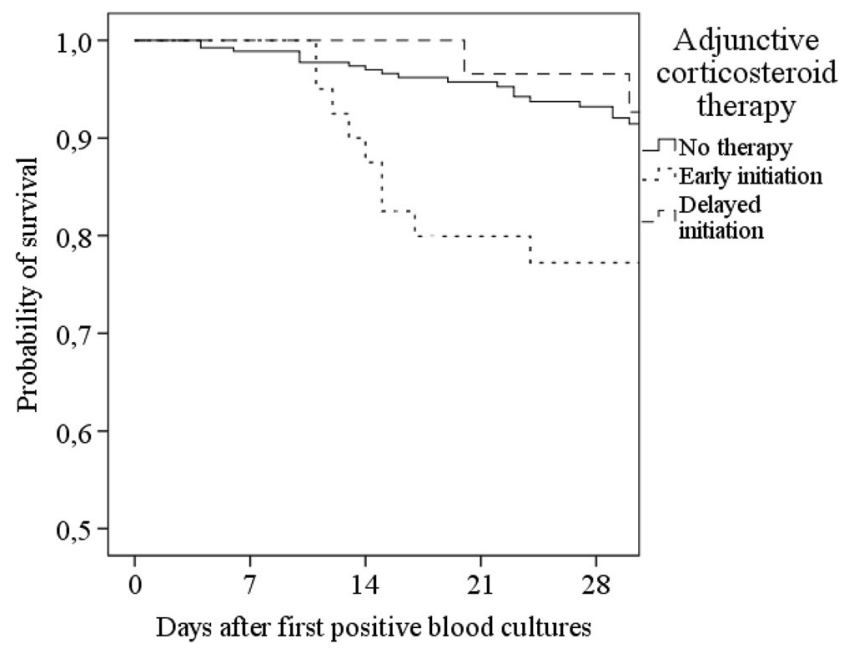

Fig. 2 Kaplan-Meier analysis of 28-day survival of 340 Staphylococcus aureus bacteremia patients categorized according to corticosteroid therapy (log-rank 0.04). Patients with corticosteroid therapy equivalent to prednisone $\geq 10 \mathrm{mg} /$ day for $\geq 1$ month prior to positive blood culture and all patients with severe sepsis or septic shock at blood culture collection time-point were excluded $(\mathrm{N}=69)$. No cases of methicillinresistant $S$. aureus were included $(\mathrm{N}=6)$. Corticosteroid therapy: Early initiation, i.e. within week 1 of positive blood culture for $S$. aureus; Delayed initiation within weeks 2-4 of positive blood culture; Late initiation within weeks 5-12 of positive blood culture

\section{Discussion}

The main finding of our study was a significantly poorer outcome among SAB patients receiving early initiated corticosteroid therapy as compared to those treated without corticosteroid therapy or with corticosteroid therapy at a later stage. Patients treated with early initiated corticosteroid therapy had a more than 3-fold higher hazard ratio for a fatal outcome when adjusting for all prognostic factors.

Parameters with indisputable prognostic impact in $\mathrm{SAB}$ are immunosuppression, severity of illness at blood culture collection time-point and proper antibiotic therapy $[4,22,33]$. Immunosuppressive therapy (including corticosteroid therapy) prior to positive blood culture is known to deteriorate the prognosis of SAB [3-6, 22]. SAB patients suffering from severe sepsis or septic shock present with high mortality $[4,7$, 22] and systemic corticosteroids are widely applied as sepsis therapy despite controversial results in large clinical trials. Furthermore, prognosis of SAB is impaired by delayed proper antibiotic therapy [33]. MRSA is connected to delayed effective antibiotic therapy and poorer outcome $[6,9,34]$ and vancomycin therapy is known to increase the risk for persistent and recurrent $\mathrm{SAB}$ and to impair prognosis as compared to beta-lactam antibiotic therapy $[35,36]$. In the present study patients with early corticosteroid therapy received significantly more often vancomycin therapy as compared to patients treated without corticosteroid therapy or patients with delayed corticosteroid therapy. However, altogether only 8 (2\%) patients received vancomycin therapy and the low n-number did not allow for reliable statistical analyses. Thus, we were not able to evaluate the prognostic impact of vancomycin treatment among patients with corticosteroid therapy.

This study excluded patients with corticosteroid therapy equivalent to prednisone $\geq 10 \mathrm{mg} /$ day for $\geq 1$ month prior to positive blood culture results and included only hemodynamically stabile patients. No MRSA cases were included and all patients were provided with an effective antibiotic therapy from the time-point of the first positive blood culture and only 
Table 3 Cox proportional hazards regression model for prognostic factors of 28-day mortality in S. aureus bacteremia patients $(\mathrm{N}=340)$

\begin{tabular}{|c|c|c|c|c|}
\hline Variable & Univariate analysis OR $(95 \% \mathrm{CI})$ & p-value & Multivariate analysis HR $(95 \% \mathrm{CI})$ & p-value \\
\hline Age $>60$ years & $11.1(3.31-37.4)$ & $<0.001$ & $10.1(2.93-34.8)$ & $<0.001$ \\
\hline Nosocomial bacteremia & $0.96(0.45-2.02)$ & NS & & \\
\hline Healthy-nonfatal underlying disease ${ }^{a}$ & $0.16(0.08-0.36)$ & $<0.001$ & $0.30(0.14-0.65)$ & 0.002 \\
\hline Alcoholism & $3.52(1.45-8.57)$ & 0.003 & $3.25(1.36-7.79)$ & 0.008 \\
\hline Any malignancy & $2.42(0.97-6.02)$ & NS & & \\
\hline Rifampicin therapy $>14$ days ${ }^{b}$ & $0.27(0.11-0.67)$ & 0.003 & $0.24(0.09-0.61)$ & 0.003 \\
\hline Endocarditis & $2.47(1.07-5.70)$ & 0.03 & $4.49(1.94-10.4)$ & $<0.001$ \\
\hline Pneumonia & $2.93(1.37-6.30)$ & 0.004 & & \\
\hline $\begin{array}{l}\text { Corticosteroid, early initiation }{ }^{\mathrm{c}} \\
\text { Corticosteroid, delayed initiation }^{\mathrm{d}}\end{array}$ & $\begin{array}{l}3.39(1.39-8.25) \\
0.74(0.17-3.28)\end{array}$ & $\begin{array}{l}0.005 \\
\text { NS }\end{array}$ & $3.75(1.60-8.79)$ & 0.002 \\
\hline
\end{tabular}

Originally altogether $430 \mathrm{SAB}$ patients were identified. Patients with corticosteroid therapy equivalent to prednisone $\geq 10 \mathrm{mg} /$ day for $\geq 1 \mathrm{month}$ prior to positive blood culture and all patients with severe sepsis or septic shock at blood culture collection time-point were excluded $(\mathrm{N}=69)$. Patients were grouped according to time-point of corticosteroid treatment initiation: 40 patients had early corticosteroid initiation, 31 patients had delayed corticosteroid initiation and 21 patients had late corticosteroid initiation. Patients with late corticosteroid initiation were not included in the analyses. Hazards ratio (OR) and $95 \%$ confidence intervals $(95 \% \mathrm{CI})$ are presented. $N S$ non-significant

a According to McCabe and Jackson [31]

b Additional antibiotic therapy

${ }^{\mathrm{c}}$ Corticosteroid therapy initiation within week 1 of positive blood culture for $S$. aureus

${ }^{\mathrm{d}}$ Corticosteroid therapy initiation within week 2-4 of positive blood culture for $S$. aureus

$2 \%$ received vancomycin. Hence, we were able to analyze the impact of corticosteroid therapy in hemodynamically stabile SAB without any external interfering corticosteroid therapy and without any bias or delay in proper antibiotic therapy onset.

However, our study has several weaknesses which have to be taken into account when the results are interpreted. Although the patients were followed prospectively they were not randomized according to corticosteroid treatment. Complicated pulmonary diseases and pneumonia in association with SAB has been described as a powerful parameter for poor outcome [3, 22, 37, 38]. This was observed also in the present study as patients with early corticosteroid treatment due to pulmonary complications demonstrated very high (63\%) mortality rates.

It may be argued that patients with early initiated corticosteroid therapy had a more severe form of SAB, including a more pessimistic prognosis initially, and thus were more prone to receive corticosteroid therapy at an early phase. To further investigate this argument a Cox proportional hazards regression analysis was performed including only patients with chronic pulmonary disease or SAB related pneumonia and within this subgroup of patients early initiated corticosteroid therapy had an evident negative prognostic impact which was further accentuated when including only patients receiving early initiated corticosteroid therapy with a minimum of prednisone $100 \mathrm{mg} /$ week. Another major bias is the low number of patients with early corticosteroid therapy in the various indication groups not making it possible to analyze them with statistical testing. However, as corticosteroids are not generally indicated for SAB treatment, randomized studies would not be ethically justifiable. We think that our study, despite it's weaknesses, has a clear message to clinicians to overweigh the indications of corticosteroid treatment carefully in the early treatment of SAB.

Various attempts were made to control reasons for the differences in outcome between patients with early and delayed initiation of corticosteroid therapy and patients treated without corticosteroid therapy. Differences in age, bacteremia acquisition, underlying conditions or antibiotic therapy did not explain the poorer prognosis. Patients with deep infection foci, including pneumonia and osteomyelitis, were more prone to receive early initiated corticosteroid therapy as compared to patients treated without corticosteroid therapy. Parameters with independent prognostic impact have been identified in earlier studies, e.g. age $>60-65$ years $[1,38]$, severe underlying diseases [1,36], adjunctive rifampicin therapy [30], chronic alcoholism [4, 22] and endocarditis [1, 22]. When controlling for all of these parameters early initiated corticosteroid therapy remained an independent prognostic marker for 28 and 90 days fatal outcome.

The exclusion of patients with corticosteroid therapy prior to $\mathrm{SAB}$ diagnosis and the inclusion of only hemodynamically stabile patients have inevitably influenced the results as immunosuppressive therapy, severe sepsis and septic shock associate to high mortality $[4,7,22,33]$. Furthermore, each 
patient was provided with a formal infectious disease specialist consultation which is known to improve treatment results and prognosis $[1,3,39]$. Altogether, these factors have most probably contributed to an overall lower mortality of only $8 \%$ at 28 days and of $12 \%$ at 90 days as compared to earlier studies presenting 28-30 days and 90 days mortality figures of $16-26 \%$ [5, 38-40] and 12-32\%, respectively [1, 2]. However, although the overall mortality in the present study was low, a negative prognostic impact due to early corticosteroid treatment was evident.

In conclusion, our data suggests that in hemodynamical stabile SAB early initiation of corticosteroid therapy may be associated to poor outcome as compared to delayed initiation of corticosteroid therapy or no corticosteroid therapy. The authors encourage caution regarding early use of corticosteroids in the management of hemodynamical stabile SAB patients.

\section{Compliance with ethical standards}

Conflict of interest The authors declare that they have no conflict of interest.

\section{References}

1. Rieg S, Peyerl-Hoffmann G, de With K et al (2009) Mortality of S. aureus bacteremia and infectious diseases specialist consultation-a study of 521 patients in Germany. J Infect 59(4):232-239

2. Pragman AA, Kuskowski MA, Abraham JM et al (2012) Infectious disease consultation for staphylococcus aureus bacteremia improves patient management and outcomes. Infect Dis Clin Pract 20(4):261-267

3. Forsblom E, Ruotsalainen E, Ollgren J, Järvinen A (2012) Telephone consultation cannot replace bedside infectious disease consultation in the management of Staphylococcus aureus bacteraemia. Clin Infect Dis 56(4):527-535

4. Kaech C, Elzi L, Sendi P et al (2006) Course and outcome of Staphylococcus aureus bacteraemia: a retrospective analysis of 308 episodes in a Swiss tertiary-care centre. Clin Microbiol Infect 12(4):345-352

5. Hill PC, Birch M, Chambers S et al (2001) Prospective study of 424 cases of Staphylococcus aureus bacteraemia: determination of factors affecting incidence and mortality. Intern Med J 31(2):97-103

6. Choi SH, Cho SY, Park JH, Chung JW (2011) Impact of infectiousdisease specialist consultations on outcomes of Staphylococcus aureus bacteremia in a hospital with a low volume of patients with $\mathrm{S}$. aureus bacteremia. J Infect 62(2):181-185

7. Ammerlaan H, Seifert H, Harbarth S et al (2009) Adequacy of antimicrobial treatment and outcome of Staphylococcus aureus bacteremia in 9 Western European countries. Clin Infect Dis 49(7): 997-1005

8. Fowler VG Jr, Olsen MK, Corey GR et al (2003) Clinical identifiers of complicated Staphylococcus aureus bacteremia. Arch Intern Med 163(17):2066-2072

9. Cosgrove SE, Sakoulas G, Perencevich EN, Schwaber MJ, Karchmer AW, Carmeli Y (2003) Comparison of mortality associated with methicillin-resistant and methicillin-susceptible
Staphylococcus aureus bacteremia: a meta-analysis. Clin Infect Dis 36(1):53-59

10. Snyder DS, Unanue ER (1982) Corticosteroids inhibit murine macrophage Ia expression and interleukin-1 production. J Immunol 129(5):1803-1809

11. Parant M, Le Contel C, Parant F, Chedid L (1991) Influence of endogenous glucocorticoid on endotoxin-induced production of circulating TNF-alpha. Lymphokine Cytokine Res 10(4):265-71

12. Schumer W (1976) Steroids in the treatment of clinical septic shock. Ann Surg 184(3):333-341

13. Bone RC, Fisher CJ Jr, Clemmer TP, Slotman GJ, Metz CA, Balk RA (1987) A controlled clinical trial of high-dose methyl-prednisolone in the treatment of severe sepsis and septic shock. N Engl J Med 317(11):653-658

14. Sprung CL, Caralis PV, Marcial EH et al (1984) The effect of highdose corticosteroids in patients with septic shock. N Engl J Med 311(18):1137-1143

15. Lefering R, Neugebauer EAM (1995) Steroid controversy in sepsis and septic shock: a meta-analysis. Crit Care Med 23(7):1294-1303

16. Cronin L, Cook DJ, Carlet J et al (1995) Corticosteroid treatment for sepsis: a critical appraisal and meta-analysis of the literature. Crit Care Med 23(8):1430-1439

17. Bollaert PE, Charpentier C, Levy B, Debouverie M, Audibert G, Larcan A (1998) Reversal of late septic shock with supraphysiologic doses of hydrocortisone. Crit Care Med 26(4): 645-650

18. Annane D, Sebille V, Charpentier C, Bollaert PE, Francois B, Korach JM et al (2002) Effect of treatment with low doses of hydrocortisone and fludrocortisone on mortality in patients with septic shock. JAMA 288(7):862-871

19. Annane D, Bellissant E, Bollaert PE, Briegel J, Keh D, Kupfer Y (2004) Corticosteroids for severe sepsis and septic shock: a systematic review and meta-analysis. BMJ 329(7464):480-488

20. Annane D, Bellissant E, Bollaert PE, Briegel J, Keh D, Kupfer Y (2004) Corticosteroids for treating severe sepsis and septic shock. Cochrane Database Syst Rev 1:CD002243

21. Moran JL, Graham PL, Rockliff S, Bersten AD (2010) Updating the evidence for the role of corticosteroids in severe sepsis and septic shock: a Bayesian meta-analytic perspective. Crit Care 14(4):R134

22. Forsblom E, Ruotsalainen E, Mölkänen T, Ollgren J, Lyytikäinen O, Järvinen A (2011) Predisposing factors, disease progression and outcome in 430 prospectively followed patients of healthcare- and community-associated Staphylococcus aureus bacteraemia. J Hosp Infect 78(2): 102-107

23. Walters JA, Tan DJ, White CJ, Gibson PG, Wood-Baker R, Walters EH (2014) Systemic corticosteroids for acute exacerbations of chronic obstructive pulmonary disease. Cochrane Database Syst Rev 1(9):CD001288

24. van der Goes MC, Jacobs JW, Bijlsma JW (2014) The value of glucocorticoid co-therapy in different rheumatic diseases - positive and adverse effects. Arthritis Res Ther 13(16)Suppl 2):S2

25. Berghauser Pont LM, Dammers R, Schouten JW, Lingsma HF, Dirven CM (2012) Clinical factors associated with outcome in chronic subdural hematoma: a retrospective cohort study of patients on preoperative corticosteroid therapy. Neurosurgery 70(4):873880

26. Dran G, Berthier F, Fontaine D, Rasenrarijao D, Paquis P (2007) Effectiveness of adjuvant corticosteroid therapy for chronic subdural hematoma: a retrospective study of 198 cases. Neurochirurgie 53(6):477-482

27. Yiannopoulou KG, Floudas CS, Efthymiou AE, Karydakis KD (2008) Reversal of tetraparesis due to staphylococcal cervical spondylodiscitis associated with anterior epidural abscess after conservative treatment. Acta Neurochir 150(9):927-928

28. McKinsey DS, McMurray TI, Flynn JM (1990) Immune complex glomerulonephritis associated with Staphylococcus aureus 
bacteremia: response to corticosteroid therapy. Rev Infect Dis 12(1):125-127

29. Ruotsalainen E, Järvinen A, Koivula I et al (2006) Finlevo Study Group: Levofloxacin does not decrease mortality in Staphylococcus aureus bacteraemia when added to the standard treatment: a prospective and randomized clinical trial of 381 patients. J Intern Med 259(2):179-190

30. Forsblom E, Ruotsalainen E, Järvinen A (2015) Improved outcome with early rifampicin combination treatment in methicillin-sensitive Staphylococcus aureus bacteraemia with a deep infection focus - $\mathrm{a}$ retrospective cohort study. PLoS One 13:10:e122824

31. McCabe WR, Jackson GG (1962) Gram negative bacteraemia. Etiology and ecology. Arch Intern Med 110:847-855

32. Levy MM, Fink MP, Marshall JC et al (2003) SCCM/ESICM/ ACCP/ATS/SIS: $2001 \mathrm{SCCM} / \mathrm{ESICM} / \mathrm{ACCP} / \mathrm{ATS} / \mathrm{SIS}$ International sepsis definitions conference. Crit Care Med 31(4): $1250-1256$

33. Lodise TP, McKinnon PS, Swiderski L, Rybak MJ (2003) Outcomes analysis of delayed antibiotic treatment for hospitalacquired Staphylococcus aureus bacteremia. Clin Infect Dis 36(11):1418-1423

34. Pfaller MA, Jones RN, Doern GV, Sader HS, Kugler KC, Beach ML (1999) Survey of blood stream infections attributable to grampositive cocci: frequency of occurrence and antimicrobial susceptibility of isolates collected in 1997 in the United States, Canada, and Latin America from the SENTRY Antimicrobial Surveillance Program. SENTRY-Participants Group. Diagn Microbiol Infect Dis 33(4):283-297

35. Siegman-Igra Y, Reich P, Orni-Wasserlauf R, Schwartz D, Giladi M (2005) The role of vancomycin in the persistence or recurrence of Staphylococcus aureus bacteraemia. Scand J Infect Dis 37(8):572578

36. Kim SH, Kim KH, Hb K et al (2008) Outcome of vancomycin treatment in patients with methicillin-susceptible Staphylococcus aureus bacteremia. Antimicrob Agents Chemother 52(1):192-197

37. Conterno LO, Wey SB, Castelo A (1998) Risk factors for mortality in Staphylococcus aureus bacteremia. Infect Control Hosp Epidemiol 19(1):32-37

38. Mylotte JM, Tayara A (2000) Staphylococcus aureus bacteremia: predictors of 30-day mortality in a large cohort. Clin Infect Dis 31(5):1170-1174

39. Honda H, Krauss MJ, Jones JC et al (2010) The value of infectious diseases consultation in Staphylococcus aureus bacteremia. Am J Med 123(7):631-637

40. Nagao M, Iinuma Y, Saito T et al (2009) Close cooperation between infectious disease physicians and attending physicians results in better outcomes for patients with Staphylococcus aureus bacteraemia. Clin Microbiol Infect 16(12):1783-1788 